\title{
Innenansichten und Perspektiven der kaufmännischen Berufsfamilie
}

Die Tradition der kaufmännischen Berufe ist lang und gekennzeichnet von zunehmender Ausdifferenzierung, zuletzt durch die Spezialisierung in der Dienstleistungswirtschaft. Dennoch gibt es keine aktuelle Untersuchung darüber, was das „Kaufmännische“ ausmacht, worin die gemeinsamen Qualifikationen dieser Berufsfamilie liegen bzw. wodurch sich die ihr zugehörigen Berufe unterscheiden. Dies ist umso erstaunlicher, da die Nachfrage nach kaufmännischen Qualifikationen und damit die Relevanz dieser Berufe deutlich zugenommen hat. Ein jüngst abgeschlossenes Forschungsprojekt des Bundesinstituts für Berufsbildung (BIBB) hat in den vergangenen vier Jahren versucht, zur Schließung dieser Forschungslücke beizutragen.

RAINER BRÖTZ, FRANZ KAISER

\section{Kaufmännische Berufe: historischer Vergleich und aktuelle Entwicklungen}

Mit über 652.000 Ausbildungsverhältnissen (BIBB 2013) entfallen auf die kaufmännischen Berufe fast $45 \%$ aller Ausbildungsverhältnisse im dualen Berufsbildungssystem. Um die kaufmännische Berufsfamilie näher zu beleuchten, soll in diesem Beitrag folgenden Fragestellungen nachgegangen werden: Wo liegen die Wurzeln der kaufmännischen Berufe und wie haben sie sich bis heute weiterentwickelt? Was sind die Kernqualifikationen und Unterschiede der kaufmännischen Aus- und Fortbildungsregelungen? Wie lässt sich die kaufmännische Berufsfamilie beschreiben und welche Anforderungen stellen sich an die künftigen kaufmännischen Berufe? ${ }^{1}$

Die skizzierten Forschungsfragestellungen sind in mehrfacher Hinsicht praxisrelevant: Auf Grundlage der gesuchten Erkenntnisse lassen sich erstens die Möglichkeiten besser ausloten, wie unterschiedliche kaufmännische Berufe im Hinblick auf eine wohnortnahe Beschulung in der Berufsausbildung neu gruppiert werden können. Ein ähnlicher Bedarf besteht mit Blick auf die demografisch bedingten Veränderungen der Ausbildungsnachfrage. Zweitens lässt sich die Neuordnungsarbeit effizienter gestalten, wenn auf standardisierte Qualifizierungsteile (Berufsbildpositionen) für die kaufmännischen Ausbildungsberufe zurückgegriffen werden kann. Zudem erleichtert ein systematischer Einblick in die Ausbildungsinhalte die Berufswahl, die wechselseitige Anerkennung von Ausbildungsteilen, die systematische Weiterentwicklung des kaufmännischen Unterrichts an den berufsbildenden Schulen und ggf. die Anrechnung auf akademische Bildungsgänge insbesondere bei Fortbildungsberufen. Und schließlich lässt sich notwendiger Reformbedarf in den (kaufmännischen) Berufen aus gewerkschaftlicher Perspektive nur dann exakt bestimmen, wenn ein Überblick über die Inhalte in den aktuellen Ausbildungsberufen vorhanden ist und zugleich die derzeit relevanten Herausforderungen kaufmännischer Facharbeit deutlich sind. (s. Abschnitt 4 und 5).

Die kaufmännischen Berufe haben eine lange, bisher in der wissenschaftlichen Diskussion wenig beachtete Tradition, die im Rahmen des GUK-Forschungsprojektes ausgeleuchtet

1 Der Beitrag stützt sich auf die Ergebnisse des BIBB - Forschungsprojektes "Gemeinsamkeiten und Unterschiede kaufmännisch-betriebswirtschaftlicher Berufe" (kurz GUK, vgl. Brötz et al. 2013), in dem neben umfangreichen Analysen aller kaufmännisch-betriebswirtschaftlichen Aus- und Fortbildungsordnungen (Ordnungsmittelanalyse) eine Literaturstudie zur Geschichte der Kaufleute (Reinisch 2011) und eine soziologische Studie erstellt wurden, die die berufliche Stellung der Kaufleute zwischen Angestelltenstatus und Dienstleistungsarbeit untersucht (Haipeter 2011). Schließlich wurde mit dem Basisdatensatz der BIBBBAuA-Erwerbstätigenbefragung 2011/2012 (Hall 2011) eine Zusatzbefragung bei kaufmännisch Tätigen durchgeführt. 
worden ist und insbesondere für das Verständnis der gemeinsamen „Familiengeschichte“ kaufmännischer Berufe hilfreich ist. Die Kaufleute im frühen Mittelalter befanden sich in keiner komfortablen Situation. Ihre kaufmännische Tätigkeit hatte ein geringes Ansehen und gehörte zu den gebrandmarkten Berufen, weil sie in Verbindung zu drei der sieben Todsünden wie Geiz, Völlerei und Faulheit gesehen wurden (Reinisch 2011, S. 46). Ferner war die Schriftsprache lateinisch - eine Sprache, deren Verbreitung und Unterrichtung der Kirche vorbehalten war. Die Händler benötigten aber die deutsche Sprache oder die Sprache der Länder, mit denen sie Handel betrieben, um die für ihre Geschäfte relevanten Informationen austauschen zu können (Kaiser 2013). ${ }^{2}$

In historischer Perspektive kann unterschieden werden zwischen den Hökern, als wandernde Kaufleute, den Krämern, als ortsfeste Kleinhändler des Detailhandels sowie den Großkaufleuten und Patriziern, die mit Fuhrwerken und Schiffen Handel über größere Entfernungen betrieben. Ihnen gemeinsam war die Selbstständigkeit als Kaufmann. Eng verknüpft mit dem Warenhandel war der Transport zur Versorgung der Menschen mit Lebensmitteln, Kleidung und Rohstoffen. Wurde der Transport zunächst von den Kleinhändlern noch selbst organisiert, so gab es parallel für jene, die es sich leisten konnten, bereits Helfer: Fuhrleute mit Pferdekutschen und Seefahrer mit Schiffen, die den Transport übernahmen. Zum Warenverkehr entwickelte sich auch der Geldverkehr als anerkanntes Tauschmittel, und damit bildete sich das Bankensystem in seiner Frühform heraus. Verbunden mit der gesellschaftlichen Arbeitsteilung entwickelte sich auch die Teilung der beruflichen Arbeiten und in deren Folge die Ausdifferenzierung der kaufmännischen Tätigkeiten.

Über lange Zeit war in der Qualifikationsentwicklung der Kaufleute die betriebliche Lehre dominant. Während die einfachen Kaufleute ihr Wissen vorrangig an ihre Kinder weitergaben, schickten die Patrizier ihre Kinder ins Ausland (z.B. nach Venedig), damit sie dort kaufmännische Erfahrungen sammelten und Fremdsprachen lernten. Im Zentrum der Ausbildung stand das Erlernen des Umgangs mit Waren, Verträgen, Lagerung und Transport, Sprachen, räumlichen Gegebenheiten und Vorschriften auf Märkten, Zöllen, Abgaben sowie Buchung, Rechnungslegung und Finanzierung. Hinweise auf diese zentralen Inhalte kaufmännischer Bildung lassen sich aus Briefwechseln herauslesen, die viele Parallelen zu aktuellen Inhalten aufweisen (vgl. dazu auch Abschnitt 4, Übersicht 1). Erst sehr spät erfolgt die Einrichtung von staatlichen kaufmännischen Schulen in Deutschland, und deutlich später als im Handwerk findet eine Ausdifferenzierung der Qualifikationsprofile der Berufe statt.

Die Preußische Gewerbeordnung und die Gewerbeordnung des Norddeutschen Bundes 1869 sind erste Meilensteine der Regelung der betrieblichen Lehre und verweisen auf Lehrverträge und Unterweisungen, ohne aber die Inhalte der Berufsausbildung näher festzulegen. Dies blieb dem Lehrherren selbst überlassen. Erst Ende der 1930er Jahre wurden staatliche Berufe für kaufmännische Ange-

\section{TABELLE 1}

\section{Konzentration der kaufmännischen Ausbildungsverhältnisse}

Angaben in absoluten Zahlen

\begin{tabular}{l|c|c|c}
\hline \multirow{2}{*}{ Berufsbezeichnung } & \multicolumn{2}{|c}{ Ausbildungsverhältnisse } \\
\cline { 2 - 4 } & $\mathbf{1 9 7 4 ^ { 1 }}$ & $\mathbf{1 9 9 3 ^ { 2 }}$ & $\mathbf{2 0 1 1}^{\mathbf{3}}$ \\
\hline 1. Industriekaufmann/-frau & 78.800 & 57.858 & 51.045 \\
\hline 2. Verkäufer/-in & 75.807 & 20.130 & 48.699 \\
\hline 3. Kaufmann/-frau im Groß- und Außenhandel & 57.072 & 49.137 & 38.811 \\
\hline 4. Bankkaufmann/-frau & 54.753 & 66.324 & 35.823 \\
\hline 5. Bürokaufmann/-frau & 46.147 & 64.836 & 52.167 \\
\hline 6. Kaufmann/-frau im Einzelhandel & 40.447 & 73.776 & 67.854 \\
\hline Summe 1-6 & 353.026 & 332.061 & 294.399 \\
\hline Gesamt kaufmännische Berufsfamilie ${ }^{4}$ & - & $534.951^{*}$ & 652.025 \\
\hline Auszubildende insgesamt ${ }^{5}$ & - & 1.629 .312 & 1.460 .658 \\
\hline $\begin{array}{l}\text { Auszubildende insgesamt abzügl. } \\
\text { der kaufmännischen Berufe }\end{array}$ & - & 1.094 .361 & 808.633 \\
\hline
\end{tabular}

* Bei dieser Zahl ist zu berücksichtigen, dass 199318 Berufe der aktuell definierten kaufmännischen Berufsfamilie noch nicht existierten.

Quelle:

Kutscha (1976), S. 44

"Datenbank Auszubildende" des BIBB auf Basis der Daten der Berufsbildungsstatistik

der statistischen Ämter des Bundes und der Länder (Erhebung zum 31. Dezember);

Statistisches Bundesamt (2012), S. 28;

nach den GUK-Kriterien, vgl. Brötz et al. (2013);

BIBB (2013), S. 121

stellte erlassen und es entstanden Verordnungen für die Industriekaufleute (1936), Einzelhandelskaufleute (1940) und Verkäufer (1941). In den Jahren danach bis 1974 wuchs die Zahl der kaufmännischen Berufe auf ca. 24 Ausbildungsberufe an (Kutscha 1976, S. 44) und umfasste dann ca. 41 Berufe im Berufsfeld Wirtschaft und Verwaltung im Jahr 1985, die einen starken kaufmännischen Zuschnitt aufweisen (BIBB 1985). Nach aktuellen Analysen des BIBB umfasst die kaufmännische Berufsfamilie 54 Ausbildungsberufe, ${ }^{3}$ worauf im Abschnitt 3 noch eingegangen wird. Allerdings ist, wie Tabelle 1 zeigt, die quantitative Dominanz von sechs Berufen auffällig.

2 Im frühen Mittelalter konnten viele Kaufleute oftmals weder lesen noch schreiben (Reinisch 2011, 22f.). Daraus erklärt sich, warum auf Schreiber zurückgegriffen wurde, die die Abwicklung des Schriftverkehrs übernahmen, oder Dolmetscher, die die Übersetzung in das und aus dem Lateinischen oder in fremde Sprachen besorgten. Nicht selten waren dies Mönche, die der lateinischen und deutschen Sprache in Wort und Schrift mächtig waren. Hieraus erklärt sich auch die Übersetzung des Kaufmanns ins Englische mit clerk.

3 Da Berufe Übereinstimmungen in ihren Fertigkeiten, Kenntnissen und Fähigkeiten aufweisen, lassen sich daraus anhand ihrer gemeinsamen und typischen Qualifikationen Gruppen bilden. Die gemeinsamen Qualifikationen der einzelnen Berufe sowie ihr Status und ihre Mentalität konstituiert sie als 
Der Anteil der Ausbildungsverhältnisse der sechs häufigsten kaufmännischen Berufe an allen Ausbildungsverhältnissen in den kaufmännischen Berufen beträgt 45,2 \% mit abnehmender Tendenz, weil neue Dienstleistungsberufe Akzeptanz im Ausbildungsmarkt finden. Der Anteil der kaufmännischen Berufsfamilie (54 Berufe) mit 652.025 Ausbildungsverhältnissen gegenüber allen übrigen 808.633 Ausbildungsverhältnissen beträgt 44,6 \% mit steigender Tendenz. Setzt man die Gesamtzahl von ca. 345 Berufen zu den 54 kaufmännischen Berufen ins Verhältnis, dann zeigt sich, dass ca. $14 \%$ der Ausbildungsberufe fast $50 \%$ aller Ausbildungsverhältnisse umfassen und zugleich in allen relevanten Wirtschaftszweigen und Sektoren zu finden sind.

Kaufleute im Einzelhandel und Verkäufer bzw. Verkäuferinnen stellen im Jahr 2011 zusammen mit 119.076 Ausbildungsverhältnissen den größten Branchenberuf im kaufmännischen Bereich. Den größten branchenübergreifenden Beruf bilden die drei Büroberufe ${ }^{4}$ mit derzeit insgesamt 86.238 Ausbildungsverhältnissen, die ab 2014 als ein neugeordneter Ausbildungsberuf Kaufmann/-frau für Büromanagement in Kraft treten werden (Elsner/Kaiser 2013).

In den vergangenen Jahren sind 15 neue kaufmännische Ausbildungsberufe im dualen System entstanden, in denen spezialisierte Qualifikationen bezogen auf Leistungserstellung, Beratung, Verkauf und Marketing ausgebildet werden. Diese Entwicklung spiegelt zum einen die zunehmende wirtschaftliche Bedeutung neuer Dienstleistungen auf dem Arbeitsmarkt wider. Zugleich zeigt sich hierin eine Reaktion auf den befürchteten Verlust der Ausbildungsbereitschaft in den neuen Dienstleistungsunternehmen (Walden 2007): Durch spezialisierte Berufe werden neue Anreize zur Ausbildung gesetzt, und die Verbände der neuen Dienstleistungsberufe sollen gewonnen werden, sich an der Qualifikationsentwicklung auf Ausbildungsebene zu beteiligen. In der Gesamtschau zeigt sich, dass die Mehrzahl aller Berufe Branchenberufe sind, was einen Trend unterstreicht, der mit der Entwicklung von Dienstleistungen zugenommen hat. Daneben gibt es aber auch funktional strukturierte, branchenübergreifende Berufe wie beispielsweise die Büro- und Industriekaufleute.

\section{Analyse der kaufmännischen Berufe}

Um die Fragen beantworten zu können, ob die historisch überlieferten Qualifikationen der Kaufleute noch immer die aktuellen kaufmännischen Aus- und Fortbildungsregelungen kennzeichnen und wie sich die kaufmännischen Berufe voneinander unterscheiden, wurden die Ausbildungsordnungen, Rahmenlehrpläne und Prüfungsordnungen von allen kaufmännisch-betriebswirtschaftlich affinen Berufen im GUK-Projekt analysiert. Für diese Analyse wurde ein sogenannter Codebaum entwickelt, dessen inhaltliche Struktur auf den Aus- und Fortbildungsverordnungen basiert (Brötz et al. 2013). Da nicht alle Inhalte in allen Berufen vorkamen, gliedert sich der Codebaum wie folgt:

- Obercode A: kaufmännische Gemeinsamkeiten, die bei allen kaufmännischen Berufen auftreten

- Obercode B: kaufmännische Vertiefungen und Besonderheiten, in denen sich die Berufe unterscheiden

- Obercode C: übergreifende Qualifikationen, die auch in anderen Ausbildungsberufen zu finden sind

- Obercode D: nicht-kaufmännische Qualifikationen, die sich in einzelnen Berufen bspw. auf technische Kenntnisse beziehen

Erfasst wurden darüber hinaus strukturelle Angaben wie Erlassjahr oder Beruf mit Ausdifferenzierungen (Wahlqualifikationen, Einsatzgebiete etc.). Am Ende der Codierung von Ausbildungsordnung, Rahmenlehrplan, Prüfungsordnung standen über 37.000 codierte Textstellen auf der Grundlage von über 80 Codes und Subcodes. Hatten sich nach der Zuordnung von Textbestandteilen mehrerer Ausbildungsordnungen und Rahmenlehrpläne keine weiteren Untercodes ergeben, so galt der Code als gesättigt und hinlänglich untergliedert. Dieses Vorgehen findet sich bei der qualitativen Inhaltsanalyse von Interviews in ähnlicher Form, indem man sogenannte „In-vivo-Codes“ vergibt, also zusammenfassende Überschriften für Textbestandteile, die sich aus den von den Interviewten benutzten Worten - in diesem Fall den in den Dokumenten verwendeten Begriffen - zusammensetzen (Brötz/Schapfel-Kaiser 2010, S. 27). Das Vorgehen bei der Entwicklung des Analyseinstrumentes orientierte sich am induktiven Vorgehen der qualitativen Inhaltsanalyse nach Mayring (2000).

\subsection{Gemeinsame Ausbildungsinhalte in der kaufmännischen Berufsausbildung}

$70 \%$ aller codierten Textstellen können den kaufmännischen Gemeinsamkeiten zugeordnet werden. Der Anteil der kaufmännischen Vertiefungen und Besonderheiten beträgt $13 \%$. Die übergreifenden Qualifikationen, die in den meisten modernisierten Ausbildungsberufen enthalten sind und folglich keine kaufmännische Spezifik beinhalten, haben einen Anteil von $15 \%$. Schließlich entfallen etwa $3 \%$ der Nennungen auf Qualifikationen, die sich als nichtkaufmännische Qualifikationen abgrenzen lassen.

Berufsfamilie. Die kaufmännische Berufsfamilie umfasst derzeit in Deutschland 54 Ausbildungs- und 33 Fortbildungsberufe, deren Gemeinsamkeiten und Unterschiede in der Erstaus- und Aufstiegsfortbildung inhaltsanalytisch nachgewiesen sind.

4 Dies sind: Bürokaufmann/-frau mit 52.167 Ausbildungsverhältnissen, Kaufmann/-frau für Bürokommunikation mit 31.464 Ausbildungsverhältnissen und Fachangestellte für Bürokommunikation mit 2.607 Ausbildungsverhältnissen. 
Betrachten wir nur die Gemeinsamkeiten, so bilden die nachfolgenden Kategorien den Kern der kaufmännischen Qualifikation. Die Prozentangaben geben dabei zunächst die Häufigkeiten in allen Ausbildungsordnungen an (Abbildung 1). Erst bei qualitativer Analyse der einzelnen Berufe werden die unterschiedlichen Qualifikationsniveaus und deren Anwendungsbezug deutlich.

Die Gemeinsamkeiten in den untersuchten kaufmännischen Berufsordnungen sind geprägt durch die folgenden Ausbildungsinhalte:

- „Kaufmännische Steuerung und Kontrolle“, die die Kassenführung, den Zahlungsverkehr, das kaufmännische Rechnungswesen und das Controlling beinhalten sowie Steuern, Versicherungen und andere Abgaben.

- „Absatzwirtschaft“, die den Verkauf von Waren und Dienstleistungen einschließt und die damit verbundenen Vertriebs- und Marketingaktivitäten sowie die Beratung von Kundinnen und Kunden einschließlich der Serviceleistungen und die Ausrichtung auf das Marktgeschehen beinhaltet.

- „Information und Kommunikation“, die die Verarbeitung von Informationen und Daten sowie schriftliche und mündliche Kommunikationsformen (auch Fremdsprachen) erfassen, sei es in internen Team- und Planungsgesprächen, bei Präsentationen, bei Dokumentationen oder bei der geschäftlichen Korrespondenz.

- „Recht und Vertrag“, die Kenntnisse wichtiger Rechtsvorschriften und die Berücksichtigung von rechtlichen Konsequenzen bspw. bei vertraglichen Regelungen umfassen. Die alltäglich zu berücksichtigen Rechtsvorschriften reichen dabei vom Bürgerlichen Gesetzbuch über sonderrechtliche Vorschriften in einigen Branchen, innerbetriebliche Vereinbarungen und Vorgaben bis zum Vertragsrecht.

- „Unternehmensorganisation“, zu der das sichere Agieren unter Berücksichtigung der innerbetrieblichen und unternehmensweiten Prozessketten gehört, wie auch Kenntnisse der Entscheidungswege, die die Zusammenhänge zwischen tatsächlichen Abläufen und deren Abbildungen in Übersichten, Organigrammen und Kostenplänen, aber auch die Kenntnis externer Marktbeziehungen und Geschäftspartner einschließt.

- „Volkswirtschaftliche Rahmenbedingungen“, die als externe Faktoren zu berücksichtigen sind und die das wirtschaftliche, politisch-gesellschaftliche wie auch natürliche Umfeld umfassen.

- „Personalwirtschaft“, die in der Ausbildung auf Aufgaben der Personalsachbearbeitung vorbereitet und

- „Einkauf“, der die Versorgung des Unternehmens mit Waren und Gütern sowie die Lieferantenbeziehungen umfasst.

Die Anforderungen in den einzelnen Handlungsbereichen sind in den Ausbildungsordnungen differenziert ausgeführt. So spielt im Rahmen des Handlungsbereichs "Kaufmännische Steuerung und Kontrolle“ etwa das betriebliche Rechnungswesen eine zentrale Rolle. Hier wer-
ABB. 1

\section{Gemeinsamkeiten in den Qualifikationsprofilen der kaufmännischen Ausbildungsberufe}

Angaben in Prozent

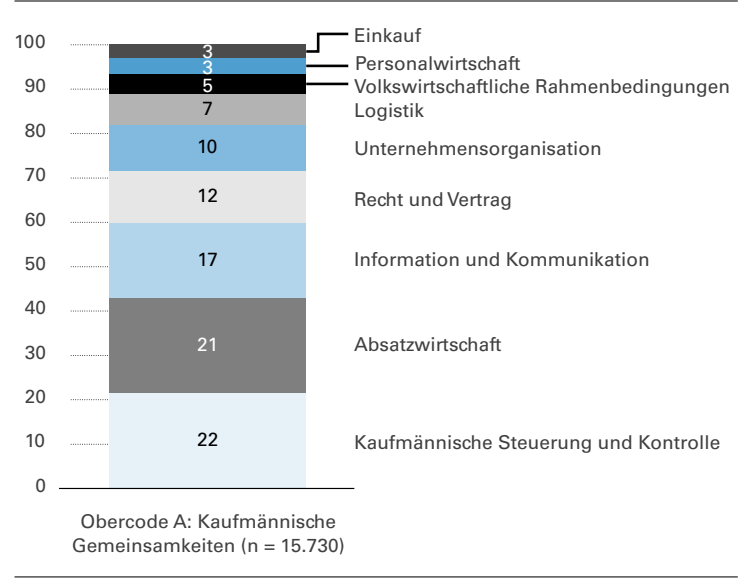

Quelle: Brötz et al. (2013).

Mitteilungen

den Qualifikationsanforderungen wie „Ermitteln der Kalkulationswerte nach der Voll- und Teilkostenrechnung, Durchführen einer Kostenstellen- und Kostenträgerrechnung“ genannt. Im Handlungsbereich „Absatzwirtschaft“ werden die Schwerpunkte auf die Kundenberatung und -orientierung sowie das Marketing gesetzt. So lauten Passagen in der Ausbildungsordnung der Automobilkaufleute: „Aufträge im Zusammenwirken mit dem Kunden formulieren und werkstattgerecht codieren.“ und „saisonale Einflüsse auf den Verkauf von Fahrzeugen, Teilen und Zubehör beachten." Hier zeigt sich exemplarisch, dass es zwar in beiden Bereiche Gemeinsamkeiten in allen analysierten Ausbildungsberufen gibt, dass sich diese grundlegenden Gemeinsamkeiten jedoch in unterschiedlicher Ausprägung - sowohl quantitativer als auch qualitativer Art - in den jeweiligen Berufen entfalten. So sind beispielsweise die Bürokaufleute im Vergleich zu den Handelsberufen weniger im unmittelbaren Kundenkontakt tätig und weisen deshalb geringere Anteile in „Absatzwirtschaft“ auf. Die Fachkraft für Lagerlogistik hat hingegen vertiefte Qualifikationsanforderungen in der Logistik.

Charakteristisch bei den Gemeinsamkeiten der Kaufleute ist, dass sie mit ihrem Handeln Situationen anderer Menschen auf der Basis betriebswirtschaftlich fundierter Wertentscheidungen beeinflussen. Sie legen Preise und Kosten fest, gestalten Arbeitsstrukturen, Verträge, beraten Kunden und verkaufen Produkte und Dienstleistungen. Deshalb erfolgte im Hinblick auf Gemeinsamkeiten auch eine genauere Betrachtung, inwiefern in diese Wertentscheidungen ethische Erwägungen oder die Berücksichtigung nachhaltiger Wirtschaftsziele eingehen. $\mathrm{Zu}$ 


\section{Kaufmännische Vertiefungen und Besonderheiten}

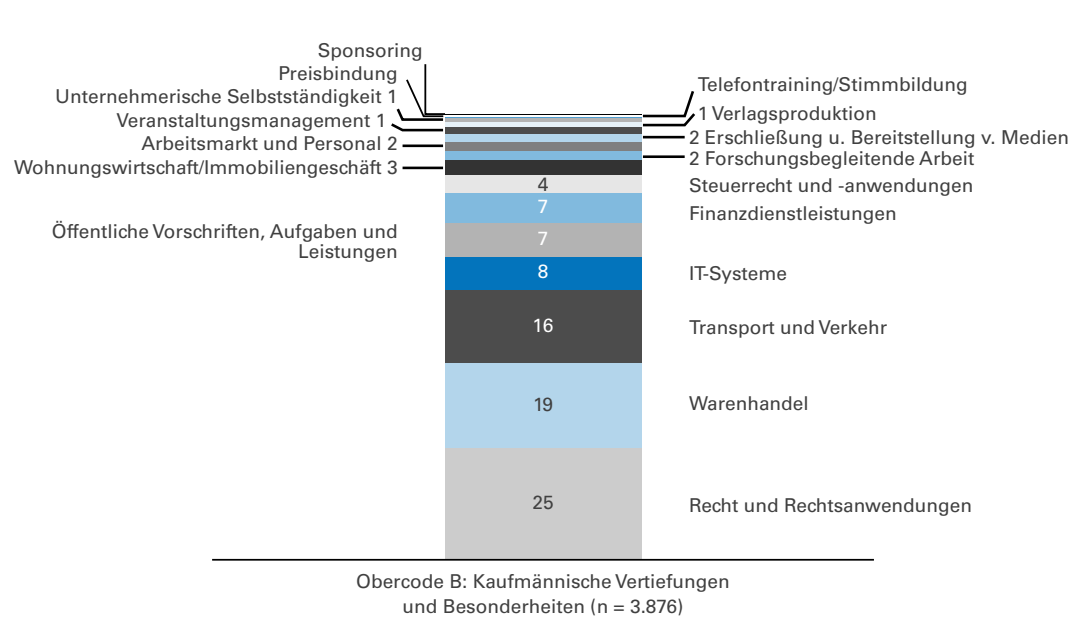

Quelle: Brötz et al. (2013). auf die jeweils zu berücksichtigenden Rechtsgebiete darstellen, die von Urheberrechten bei den Medienberufen über Haftungsrechte im Transportwesen bis zum Arbeitnehmerüberlassungsrecht bei den Personaldienstleistungskaufleuten reichen. Hierin kommt zum Ausdruck, dass die ausgebildeten Fachkräfte über unmittelbar einsetzbare, hinlänglich spezifische Qualifikationen verfügen, die auch eine hilfreiche Voraussetzung für die anschließende Facharbeitstätigkeit sind.

Die Betrachtung der kaufmännischen Vertiefungen und Besonderheiten verstärkt die zuvor schon angesprochene unterschiedliche Gewichtung der „Gemeinsamkeiten“ und prägt damit die jeweiligen Einzelprofile. Diese Prägung ist umso stärker, wenn es sich um branchenbezogene Profile handelt. Und umgekehrt schlägt sie bei den branchenübergreifenden Berufen, wie bspw. bei den Büroberufen, weniger durch.

\subsection{Kaufmännische Fortbildungsberufe}

Die Frage nach den Gemeinsamkeiten und Unterschieden der Qualifikationen stellt sich auch in den Fortbildungsordnungen. Dabei ist zu unterscheiden zwischen den kaufmännischen Angestellten, die in der Regel über eine kaufmännische oder vergleichbare Ausbildung verfügen, und den mittleren Führungskräften, die als Fachwirte und Fachkaufleute über die Fortbildungsordnungen qualifiziert werden können. Gleichfalls mit der Methode der Ausbildungsordnungsanalyse wurden auch die 33 kaufmännischen Fortbildungsordnungen analysiert. Im Unterschied zur Analyse der Ausbildungsberufe liegen hier nicht betriebliche und schulische Ausbildungspläne, sondern lediglich Prüfungsregelungen zugrunde. Als wesentliches Ergebnis konnten im Hinblick auf relevante Arbeits- und Geschäftsprozesse mittlerer Führungskräfte kaufmännischer Prägung drei berufsübergreifende Handlungsfelder identifiziert werden: die Unternehmensführung und -entwicklung, die betriebswirtschaftliche Steuerung und Kontrolle sowie die Personalführung und -entwicklung. Diese drei Handlungsfelder wurden zur Basis für ein Standardisierungskonzept im Fortbildungsbereich, das bereits in neuen Prüfungsverordnungen implementiert ist und zugleich weiterentwickelt wird (Kaiser et al. 2011). Neben den benannten drei Bereichen finden sich auf der Fortbildungsebene auch die Absatzwirtschaft sowie die Information und Kommunikation, die als kaufmännische Kernqualifikationen die Fortbildungsberufe prägen ( $A b$ bildung 3).

Ähnelt zunächst die Verteilung des Qualifikationsspektrums dem der Ausbildungsordnungen, so zeigt sich bei den Fortbildungsordnungen aber ein Bedeutungszuwachs bei den Personalaufgaben, eine Verlagerung vom buchenden Rechnungswesen zum steuernden Controlling sowie ein stärkerer Einsatz in der Unternehmensorganisation. Vergleicht man die Codierungen der Gemeinsamkeiten auf der Einzelberufsebene, so zeigen sich neben branchenspe- 
zifischen Spezialisierungen dennoch durchgehend die berufsübergreifenden Qualifikationen, die sich aus der Funktion der mittleren Führungskräfte ergeben, auf die die Fortbildungen vorbereiten.

\section{Bestimmung des „Kaufmännischen“ und der kaufmännischen Berufsfamilie}

Zur Ergänzung des Bildes von der kaufmännischen Berufsfamilie über die Inhaltsanalyse der aktuellen Berufe hinaus bietet sich ein Blick in die Soziologie, Wirtschaftstheorie, Geschichte und ins Ausland an. Exemplarisch für die Zusammenführung der unterschiedlichen Analyseergebnisse und zur Bestätigung der herausgebildeten Gemeinsamkeiten werden hier die aktuellen Qualifikationsbeschreibungen aus der historischen Literatur und die aktuellen Qualifikationsbeschreibungen der kaufmännischen Berufe in Deutschland und der Schweiz gegenübergestellt (Kock 2013). ${ }^{5}$ Einen kursorischen Überblick gibt Übersicht 1 .

Der Vergleich erhärtet die Ergebnisse der Ordnungsmittelanalyse und verdeutlicht, dass es möglich ist, Gemeinsamkeiten kaufmännischer Qualifikationen in unterschiedlichen Ländern und Zeiten auszumachen. Die Gegenüberstellung zeigt, dass Kaufleute über Kenntnisse von Waren/Dienstleistungen verfügen müssen und den Einkauf und Transport im Rahmen der Leistungserstellung organisieren und steuern. Ein Kernbereich für die Kaufleute ist die Kundenberatung und -betreuung sowie das Marketing. Das kaufmännische Rechnungswesen und die Finanzierung sind ebenfalls zentrale Handlungsbereiche. Der Einsatz von Informations- und Kommunikationstechniken zieht sich wie ein roter Faden durch die kaufmännischen Aufgaben. Sie haben die Arbeit der Kaufleute in allen Epochen mit geprägt und deren Qualifikationen beeinflusst. Aufgaben der Personalentwicklung finden sich ebenso wieder wie die der Büroorganisation und des Schriftverkehrs. Kaufleute schließen Verträge ab und müssen daher auch über nationale und zunehmend internationale Rechtsvorschriften verfügen. Letztlich sind auch die volkswirtschaftlichen Rahmenbedingungen, die früher wie heute das Handeln der Kaufleute beeinflussten, von Bedeutung. Der Vergleich mit anderen Ländern zeigt, dass von einem vergleichbaren Verständnis des „Kaufmännischen" im deutschsprachigen Raum gesprochen werden kann. $^{6}$

5 Beim Ländervergleich wurden die Bankkaufleute, Industriekaufleute und die Verwaltungsfachangestellten herangezogen.

6 Im Rahmen des Projekts wurden Vergleiche mit Österreich, der Schweiz und Schweden angestellt.
ABB. 3

\section{Häufigkeitsverteilung A-Codes bezogen auf alle 33 Fortbildungsberufe}

Angaben in Prozent

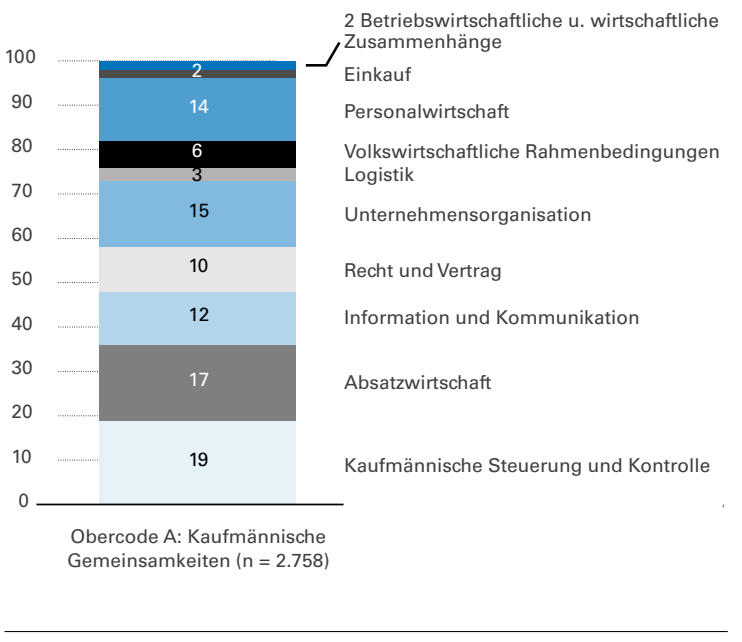

Quelle: Brötz et al. (2013).

Mitteilungen

Spiegelt man diese Gemeinsamkeiten in den Berufsordnungen zusätzlich mit den aktuellen Anforderungen, die über 2.000 kaufmännisch tätige Angestellte im Rahmen unserer Befragung im Herbst 2012 benannt haben (GUKProjekt, vgl. Fußnote 1), so entsprechen die festgestellten Gemeinsamkeiten in der kaufmännischen Ausbildung auch den gemeinsamen Anforderungen in der beruflichen Praxis (Abbildung 4). Allerdings zeigt sich eine deutliche Dominanz der kommunikativen Fähigkeiten im Alltagshandeln bei den kaufmännischen Angestellten.

\section{Aktuelle Problemlagen und Handlungsbedarf}

Aus der Ordnungsmittelanalyse, den Literaturstudien, der Befragung und den Berufsvergleichen mit anderen Ländern sowie aktuellen Diskursen in den Bezugsdisziplinen der kaufmännischen Berufsforschung zeigen sich, bezogen auf die Kernqualifikationen und Handlungssituationen kaufmännischer Angestellter, aktuelle Trends und Reformbedarfe für die künftige Neuordnungsarbeit bei den kaufmännischen Berufen. Im Rahmen der kaufmännischen Facharbeit sind grundlegende Kenntnisse im Bereich der kaufmännischen Steuerung und Kontrolle auch künftig erforderlich. Dies setzt voraus, dass nicht mehr im traditionellen Sinne gebucht wird, sondern die Steuerung der Geschäftsvorfälle als Arbeitsprozesse im Rahmen von Geschäftsprozessen verstanden 


\section{Gemeinsame Ausbildungsinhalte in der kaufmännischen Berufsfamilie im historischen und Länder- vergleich}

\begin{tabular}{|c|c|c|}
\hline $\begin{array}{l}\text { Kaufm. Qualifikationen aus } \\
\text { historischen Lehrbüchern } \\
\text { und Briefen (Reinisch 2011) }\end{array}$ & $\begin{array}{l}\text { Leit- und Richtziele der Schweizer } \\
\text { Bildungsverordnung von } 2012\end{array}$ & $\begin{array}{l}\text { Codes und Subcodes des } \\
\text { GUK-Codebaums }\end{array}$ \\
\hline $\begin{array}{l}\text { Warenkenntnisse, Warenlagerung, } \\
\text { Transportwege, Planung von } \\
\text { Transportmitteln, Handelsgeographie }\end{array}$ & $\begin{array}{l}\text { Material, Waren oder Dienst- } \\
\text { leistungen bewirtschaften }\end{array}$ & Einkauf/Logistik \\
\hline Gespräche mit Kunden & Kunden beraten & Kundenberatung/-orientierung \\
\hline $\begin{array}{l}\text { Gespräche und Verhandlungen } \\
\text { mit Kaufleuten, Fuhrleuten, } \\
\text { Transporteuren, Reedereien }\end{array}$ & Aufträge abwickeln & Auftragsabwicklung \\
\hline $\begin{array}{l}\text { Aufbauen der Stände und Waren, } \\
\text { Handelsbeziehungen über } \\
\text { Niederlassungen im Ausland }\end{array}$ & $\begin{array}{l}\text { Maßnahmen des Marketings- und } \\
\text { der Öffentlichkeitsarbeit umsetzen }\end{array}$ & $\begin{array}{l}\text { Marketing/Außenbeziehungen } \\
\text { der Unternehmen }\end{array}$ \\
\hline $\begin{array}{l}\text { Regeln und Vorschriften für Kontor-, } \\
\text { Büro und Verwaltungsangestellte }\end{array}$ & $\begin{array}{l}\text { Aufgaben der Personaladministration } \\
\text { ausführen }\end{array}$ & Personalsachbearbeitung \\
\hline $\begin{array}{l}\text { kaufmännisches Rechnen, } \\
\text { Buchhaltung, Kassenführung, } \\
\text { Kredite, Wechsel, Börse }\end{array}$ & $\begin{array}{l}\text { Finanzwirtschaftliche Prozesse } \\
\text { ausführen }\end{array}$ & $\begin{array}{l}\text { Internes Rechnungswesen/ } \\
\text { Zahlungsverkehr }\end{array}$ \\
\hline $\begin{array}{l}\text { Schreibarbeiten, Kopieren, } \\
\text { Korrespondenz, Kalligraphie, } \\
\text { "Cryptographie“(Geheimschrift) }\end{array}$ & $\begin{array}{l}\text { Administrative und organisatorische } \\
\text { Tätigkeiten ausüben }\end{array}$ & $\begin{array}{l}\text { Textverarbeitung/ } \\
\text { bürowirtschaftliche Abläufe }\end{array}$ \\
\hline $\begin{array}{l}\text { Warenkenntnisse, Warenkunde, } \\
\text { Maß- und Gewichtseinheiten, } \\
\text { Gütevorschriften }\end{array}$ & $\begin{array}{l}\text { Kenntnisse über die eigene Branche } \\
\text { und den eigenen Betrieb anwenden }\end{array}$ & $\begin{array}{l}\text { Produktkenntnisse/ } \\
\text { Warenwirtschaftssystem }\end{array}$ \\
\hline $\begin{array}{l}\text { Gespräche, Post per Kutsche } \\
\text { und Schiff }\end{array}$ & $\begin{array}{l}\text { Information, Kommunikation, } \\
\text { Administration (IKA) }\end{array}$ & Information und Kommunikation \\
\hline $\begin{array}{l}\text { Finanzwesen, Zahlungsmittel, } \\
\text { Kredite, Wechsel, Börse }\end{array}$ & Finanzwirtschaftliche Zusammenhänge & $\begin{array}{l}\text { betriebliches Rechnungswesen, } \\
\text { Finanzierung }\end{array}$ \\
\hline $\begin{array}{l}\text { Rechtskunde, Verträge, Mahnungen, } \\
\text { Umgang mit Notaren }\end{array}$ & Recht und Staat & Recht und Vertrag \\
\hline $\begin{array}{l}\text { Zölle, Steuern, Abgaben, Zoll- } \\
\text { vorschriften, staatliche Abgaben }\end{array}$ & $\begin{array}{l}\text { Gesamtwirtschaftliche und } \\
\text { gesellschaftliche Zusammenhänge }\end{array}$ & $\begin{array}{l}\text { Volkswirtschaftliche } \\
\text { Rahmenbedingungen }\end{array}$ \\
\hline
\end{tabular}

und interpretiert werden können. Hier werden den kaufmännischen Angestellten Aufgaben und Entscheidungen im Bereich des Rechnungs- und Finanzwesens zur Erfüllung von Leistungszielen im Auftrag des Unternehmens übertragen. Die Anforderung ist, den wirtschaftlichen Erfolg des Unternehmens zu sichern und zu mehren bzw. das zur Verfügung stehende Budget im öffentlichen Dienst sinnvoll auszuschöpfen. Im Kern geht es um ein grundlegendes Verständnis für die Systematik und die betrieblichen Zusammenhänge, welche für die Anwendung bzw. Nutzung beispielsweise betrieblicher Software im Bereich der kaufmännischen Steuerung und Kontrolle notwendig ist. Eine reflexive Bewertung der Wirkungen der Steuerungsinstrumente erfolgt jedoch nicht und sollte künftig zumindest auf der Fortbildungsebene Standard sein. Der Bereich der Absatzwirtschaft mit Marketing und Vertrieb sowie Kundenberatung und Kundenorientierung wird künftig neue Anforderungen an die kaufmännischen Angestellten stellen, deren berufliche Tätigkeit stark von Markt- und Kundenorientierung geprägt ist. Sie müssen verstärkt zwischen Kundenwünschen, Vorgaben des Unternehmens und eigenen Interessen balancieren und diese Situationen ausgestalten (Munz et al. 2012). Unter Nutzung ihrer sprachlichen Kompetenz werden Kunden als Ko-Produzenten von den kaufmännischen Angestellten eingebunden und als Produktentwickler an der Erstellung und Entstehung von Produkten und Dienstleistungen in Formen der „Open Innovation" beteiligt (Möslein 2009). Dabei ist die Interaktionsarbeit - unabhängig von den Segmenten und Zielgruppen - eine anspruchsvolle Tätigkeit, die fachliches Wissen über die Beschaffenheit der Güter (Waren/Dienstleistungen) vor- 
aussetzt und zugleich einem steigenden Erwartungshorizont der Kunden gegenübersteht. Um die hierfür notwendige Handlungskompetenz zu entwickeln, empfiehlt sich eine verstärkte Entwicklung kreativer Fähigkeiten in der schulischen und betrieblichen Ausbildung. Betriebswirtschaftliche Informationen werden schneller abrufbar und zugleich wird erwartet, dass sie auch schneller be- und verarbeitet werden. Dabei können soziale und materielle Räume auseinanderfallen oder miteinander verbunden werden. Die Informationsverarbeitung mit PC und mobilen Geräten und der versierte Umgang mit Schriftsprache und Symbolen werden deshalb zentrale Fähigkeiten der Kaufleute bleiben (Kaiser 2013).

Die Steigerung der Rendite bleibt bei Fortbestand der wirtschaftspolitischen Wachstumsorientierung weiterhin das Ziel von betrieblicher Reorganisation. Dabei wird der Wettbewerbs- und Kostendruck in den Unternehmen an den einzelnen Arbeitsplatz weitergeleitet. Die Finanzialisierung führt zu zentral definierten und an den Finanzmärkten orientierten Renditevorgaben mit weltweiten Auswirkungen, die nicht reflektiert werden (Chomsky 2000). Beschäftigte in Unternehmen - und dies gilt insbesondere für die kaufmännischen Angestellten - werden weniger hierarchisch, sondern eher über finanzielle Rahmenbedingen und Indikatoren geführt, die zunehmend verinnerlicht werden (Haipeter 2011). So ergibt sich ein „Siegeszug des kaufmännischen Denkens“, der die Gefahr in sich birgt, dass kaufmännische Angestellte noch stärker zu systemkonformen Multiplikatoren kapitalgetriebener Verwertungsinteressen werden. Hinsichtlich der Qualifikationsanforderungen ist zudem davon auszugehen, dass es eine Zunahme an kaufmännischen Qualifikationen im Sinne „betriebswirtschaftlicher Steuerungslogik“ in allen Ausbildungsberufen geben wird.

Um die Zusammenhänge von Marktorientierung, Datenverarbeitung, betriebswirtschaftlich-umsatzorientierter Prozessgestaltung bis hin zur sogenannten Kundenorientierung stärker aufeinander beziehen zu können, werden Kenntnisse zur Analyse der Unternehmensprozesse immer wichtiger. Diesbezüglich ist bei der Gestaltung der beruflichen Neuordnungen eine Wende erkennbar: weg von der bisherigen Betonung fachlicher Inhalte und kommunikativer Fähigkeiten hin zur Prozessorientierung in den Aus- und Fortbildungsberufen. Diese Akzentverschiebung wird insbesondere von Arbeitnehmerseite verfolgt und sie könnte, wenn dieser Trend bis in die Ausbildungsgestaltung durchschlägt, eine neue kaufmännische Bildung etablieren.

\section{Fazit}

Die historische Betrachtung hat gezeigt, dass die Wurzeln der Kaufleute in der Steuerung, Organisation und Bereitstellung von Waren, Geld und später auch von Dienstleistungen liegen, die Zahl der kaufmännischen Berufe zuge-
ABB. 4

\section{Relevante Fähigkeiten für Kaufleute im Bereich ...}

Angaben in Prozent

weiblich männlich

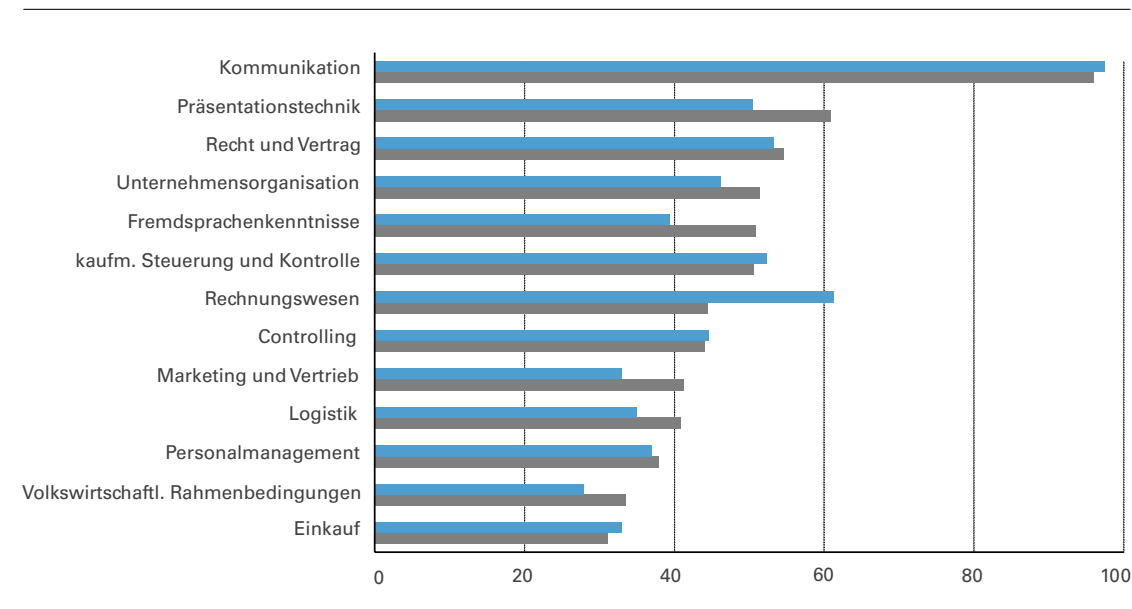

Quelle: Brötz et al. (2013).

nommen hat und sich die Berufe der kaufmännischen Angestellten im Zusammenhang mit der fortschreitenden gesellschaftlichen und betrieblichen Arbeitsteilung spezialisiert haben. Mit Hilfe der Ordnungsmittelanalyse, Literaturstudien und einer empirischen Befragung konnten die Gemeinsamkeiten ermittelt und bestätigt werden, die sich gegenwärtig für 54 kaufmännische Berufe beschreiben lassen. Diese Gemeinsamkeiten repräsentieren die Kernqualifikationen der kaufmännischen Berufsfamilie.

Mit dem GUK-Projekt (vgl. Fußnote 1) hat die ordnungsbezogene Berufsforschung einen Referenzrahmen für die Bildung von kaufmännischen Berufsgruppen vorgelegt, der im Hinblick auf Standardisierung, Gruppierung und Abgrenzung genutzt werden kann. Die Analyse hat auch gezeigt, dass auf einer hohen Aggregationsebene eine große Übereinstimmung zwischen allen kaufmännischen Berufen besteht. Die ausdifferenzierten Ergebnisse aus der Analyse einzelner Berufsgruppen innerhalb der kaufmännischen Berufsfamilie machen aber auch deutlich, dass keine vorschnellen Schlüsse im Hinblick auf die Zusammenführung von Berufen gezogen werden sollten. Solche Zusammenführungen könnten Gefahr laufen, der jeweiligen Prägung der Beruflichkeit nicht gerecht zu werden und eine Berufsbildung zu etablieren, die so allgemein bleibt, dass sie die Bezüge zu speziellen Rechtsgebieten und Handlungssituationen nicht berücksichtigt. Sie würde dann nicht zu der beruflichen Handlungsfähigkeit führen, die das deutsche Berufsbildungssystem auszeichnet. Denn erst auf der unmittelbaren Handlungsebene zeigen sich die relevanten Rahmenbedingungen, die bspw. einen Schifffahrtskaufmann vom Buchhändler unterscheiden (vgl. dazu ausführlich Brötz et al. 2013, S. 18ff.) 
Um dennoch zu einer weiteren Standardisierung der Ausbildungsordnungen im Hinblick auf Gemeinsamkeiten zu kommen, wie dies in ähnlicher Weise für die Fortbildungsordnungen geschieht, bedarf es einer berufsübergreifenden kaufmännischen Fachgruppe im BIBB, die aus Vertretern der Sozialpartner besteht. In einer solchen Gruppe könnten intendierte und nichtintendierte Wirkungen von Standards und Flexibilisierungskonzepten beurteilt und beobachtet werden. Der neue Beruf „Kaufmann/-frau für Büromanagement“, der drei zuvor bestehende Berufe mittels einer gemeinsamen Basis und darauf aufbauenden Wahlqualifikationen neu regelt, ist ein Präzedenzfall in diese Richtung und orientiert sich an den vom GUK-Projekt ausgearbeiteten kaufmännischen Gemeinsamkeiten.

Die kaum vorhandene Qualifizierung der kaufmännischen Angestellten im Hinblick auf ethische Fragestellungen und Anforderungen an ein nachhaltiges Wirtschaften weist auf uneingelöste Absichtserklärungen der Bundesregierung im Hinblick auf die systematische Verankerung der Nachhaltigkeitsthematik in der Berufsbildung hin (BMBF 2009). Dies gilt ebenso für die Inhalte der kaufmännischen Fortbildungsordnungen, die auf Positionen in der mittleren Führungsebene vorbereiten und für die derzeit eine Konzeption für berufsübergreifende Qualifikationen implementiert wird (Kaiser et al. 2011). Die hier noch vorhandenen Defizite überraschen umso mehr, da es sich um die Vorbereitung auf Führungspositionen handelt.

Betrachtet man die Aus- und Fortbildungsordnungen als Qualifikationsgrundlage und Bildungskanon für die künftigen kaufmännischen Angestellten aus der Perspektive eines gewerkschaftlichen Bildungsanspruchs zur Förderung der Persönlichkeit und eines kritikfähigen mündigen Bürgers, so vermisst man neben der Befähigung zur Reflexion wirtschaftlicher Entscheidungen unter der Berücksichtigung von Ethik, Nachhaltigkeit und Verbraucherinteressen die Entwicklung von Kreativität und Innovationsfähigkeit sowie die Fähigkeit zur Gestaltung des sozialen Miteinanders und guter Arbeit im Betrieb.

\section{LITERATUR}

Brötz, R./Annen, S./Kaiser, F./Kock, A./Noack, I./Peppinghaus, B./Schaal, T./ Tiemann, M. (2013): Abschlussbericht Forschungsprojekt „Gemeinsamkeiten und Unterschiede kaufmännisch-betriebswirtschaftlicher Aus- und Fortbildungsordnungen", Bundesinstitut für Berufsbildung, September 2013, https://www2.bibb.de/tools/fodb/pdf/eb_42202.pdf

Brötz, R./Schapfel-Kaiser, F. (2010): Gemeinsamkeiten in kaufmännischen Ausbildungsberufen ermitteln. Zwischenergebnisse einer computergestützten Dokumentenanalyse, in: Berufsbildung in Wissenschaft und Praxis 39 (4), S. $26-30$

Bundesinstitut für Berufsbildung (BIBB) (1985): Die anerkannten Ausbildungsberufe, Berlin

Bundesinstitut für Berufsbildung (BIBB) (2013): BIBB - Datenreport zum Berufsbildungsbericht 2012, Bonn

Bundesministerium für Bildung und Forschung (BMBF) (2009): Bericht der Bundesregierung zur Bildung für eine nachhaltige Entwicklung, Berlin Chomsky, N. (2000): Profit over people. Neoliberalismus und globale Weltordnung, Hamburg/Wien, download der deutschen Ausgabe: http://cdn.preterhuman.net/texts/conspiracy/Noam.Chomsky.-.Profit.Over.People.pdf Elsner, M./Kaiser, F. (2013): Interessen, Strukturen, Abläufe und Ergebnisse am Beispiel der Entwicklung des neuen kaufmännischen Allrounders -
„Kauffrau/-mann für Büromanagement“, in: Büchter, K./Bohlinger, S./Tramm, T. (Hrsg.): Ordnung und Steuerung der beruflichen Bildung, BWPAT-Heft (25), http://www.bwpat.de/content/ausgabe/25/

Haipeter, T. (2011): Kaufleute zwischen Angestelltenstatus und Dienstleistungsarbeit - eine soziologische Spurensuche. Expertise im Auftrag des GUKProjekts: BIBB, Wissenschaftliche Diskussionspapiere (126), Bonn, http://www.bibb.de/veroeffentlichungen/de/publication/show/id/6721 Hall, A. (2011): BIBB/BAuA-Erwerbstätigenbefragung 2011/2012. Projektbeschreibung, Bonn, https://www2.bibb.de/tools/fodb/pdf/at_21304.pdf Kaiser, F. (2013): Sprache als Kerninstrument des Denkens und Handelns kaufmännischer Angestellter, in: Efing, Ch. (Hrsg.): Sprachlich-kommunikative Anforderungen an Auszubildende - und ihr Verhältnis zu den realen Kompetenzen der Auszubildenden. Fachtagung Sprache der 17. Hochschultage berufliche Bildung, Sonderheft bwp@2013

Kaiser, F./Brötz, R./Annen, S./Bednarz, S./Blötz, U./Brings, C./Elsner, M./ Jordanski, G./Krämer, H./Mettin, G./Paulini-Schlottau, H./Peppinghaus, B. (2011): Konzept für berufsübergreifende Qualifikationen in kaufmännischen Fortbildungsregelungen. Abschlussbericht, Bundesinstitut für Berufsbildung, Bonn, https://www2.bibb.de/tools/fodb/pdf/eb_42344.pdf

Kock, A. (2013): Gemeinsamkeiten und Unterschiede kaufmännisch-betriebswirtschaftlicher Aus- und Fortbildungsberufe (GUK) - Kontrastierung schweizerischer Berufe, in: Wirtschaft und Erziehung 65 (4), S. 134-144

Kutscha, G. (1976): Das politisch-ökonomische Curriculum, Kronberg

Mayring, P. (2000): Qualitative Inhaltsanalyse, in: Forum Qualitative Sozialforschung (online Journal) 1 (2), Juni, http://www.qualitative-research.net/ index/php/fqs/article/view/1089/2383

Möslein, K. (2009): Innovation als Treiber des Unternehmenserfolgs. Herausforderungen im Zeitalter der Open Innovation, in: Zerfaß, A./Möslein, K. (Hrsg.): Kommunikation als Erfolgsfaktor im Innovationsmanagement, Wiesbaden

Munz C./Wagner, J./Hartmann, E. (Hrsg.) (2012): Die Kunst der guten Dienstleistungen. Wie man professionelles Dienstleistungshandeln lernen kann, Bielefeld

Reinisch, H. (2011): Geschichte der Kaufleute. Studie zur Geschichte vornehmlich der deutschen Kaufleute, ihrerTätigkeit, ihrer Stellung in der Gesellschaft sowie ihrer Organisation und Qualifizierungsstrukturen von den Anfängen bis zum Ausgang des 19. Jahrhunderts. Expertise im Auftrag des GUK-Projekt: BIBB, Wissenschaftliche Diskussionspapiere (125), Bonn

Statistisches Bundesamt (2012): Bildung und Kultur. Berufliche Bildung 2011 (Fachserie 11 Reihe 3), S. 28

Walden, G. (2007): Qualifikationsentwicklung im Dienstleistungsbereich. Herausforderungen für das duale System der Berufsbildung, Bonn

\section{AUTOREN}

RAINER BRÖTZ, war bis Oktober 2013 Arbeitsbereichsleiter im Bundesinstitut für Berufsbildung (BIBB), Bonn. Arbeitsschwerpunkte: Berufsprinzip, kaufmän nische Aus- und Fortbildungsberufe, Prüfungswesen, Strukturfragen der kaufmännischen Berufsbildung.

rainerbroetz@gmx.de

FRANZ KAISER, Dr., ist Wissenschaftlicher Mitarbeiter im BIBB, Bonn. Arbeitsschwerpunkte: Berufsforschung, kritisch-subjektorientierte Berufsbildungstheorie, kaufmännische Berufe im internationalen Vergleich.

kaiser@bibb.de 